\title{
Severe Tetanus Complicating an Open Femoral Fracture Successfully Managed Using Intrathecal Human Tetanus Immunoglobulin and External Fixation
}

\author{
Maj A Henderson, \\ BMedSci, BM BS, MRCP, RAMC \\ Maj P Guy, \\ BSc MB, FRCS, RAMC \\ Lt Col R J Knight, \\ MB BS, FFARCSI, RAMC \\ British Military Hospital, Dharan
}

SUMMARY: We report here a case of severe tetanus, complicating a compound fracture of the femur, successfully managed without intensive care facilities, using intravenous sedation and intrathecal Human Tetanus Immunoglobulin (HTI $ळ$ Substitution of conventional traction (Transtibial Steinman Pin and Thomas Splint) for a Day Frame External Fixationf $\subsetneq$ Device (EFD) provided excellent fixation and stability of the fracture in spite of severe tetanic spasms.

\section{Introduction}

Tetanus is a major health problem in the developing world, with at least 50,000 recorded fatalities each year. Prognosis is closely related to the length of the incubation period and the onset time (time between the first symptom and the first generalisation spasm). An incubation period of less than seven days or an onset time of less than 24 hours heralds a severe attack of tetanus and predicts both a poor prognosis and the need for muscle relaxation to control the spasms $^{2,3,4,5}$. Managing severe tetanus is fraught with difficulties, and even when sophisticated intensive care facilities are available the mortality can approach $40 \%{ }^{6}$. Cases of tetanus admitted to BMH Dharan, in south-east Nepal, are managed on the medical wards without the benefit of intensive care facilities although, with difficulty, assisted ventilation is possible. We describe a case of severe tetanus complicating a compound fracture of the femur, successfully managed using intravenous sedation and intrathecal human tetanus immunoglobulin.

\section{History}

A 17-year-old Nepali boy was transferred to BMH Dharan from a local hospital. Forty-eight hours earlier, he had fallen from a tree, sustaining a severely contaminated open fracture of the left femur. Procaine penicillin and tetanus toxoid had been given soon after injury and the wound dressed, although otherwise left undisturbed. He had never been immunised against tetanus. On arrival at $\mathrm{BMH}$, apart from a fever, his general condition was good, although the wound was severely infected. Intraveno⿺ metronidazole, gentamicin and cloxacillin wero commenced and extensive surgical excision of the wourg was undertaken. The fracture was initially stabilised using. a transtibial Steinman pin and Thomas splint in fixe traction. The wound was dressed with Eusol, and left open.

Three days after admission, the patient complained of abdominal discomfort and pain in the injured leg. $\stackrel{\mathbb{Z}}{\unrhd}$ Examination revealed a non-tender, rigid abdomen and $\overrightarrow{\overrightarrow{0}}$ painful spontaneous spasms of the left quadriceps. The $\frac{3}{3}$ suspicion of tetanus was confirmed a few hours later by the appearance of trismus and generalised muscular hypertonicity. He was given Human Tetanus Immunoglobulin (HTI), 250 units intramuscularly and penicillin G $600 \mathrm{mg}$, chlorpromazine $50 \mathrm{mg}$ and idazepam $50 \mathrm{mg}$ intravenously, six hourly. The following day he had well marked risus sardonicus (Figure 1) and spontaneous generalised spasms. He was given a further 250 units of HTI intravenously, and started on dexamethasone $2 \mathrm{mg}$ intravenously six hourly.

His spasm frequency rapidly increased over the next twelve hours to $20-30$ spasms per hour, despite incrementing the intravenous sedation to chlorpromazine 은 $100 \mathrm{mg}$ and valium $10 \mathrm{mg}$ four hourly. An elective $>$ tracheostomy was performed, in anticipation of respiratory 을. embarrassment. To aid nutrition, and at the same time to $\mathrm{N}$ reduce the nasogastric tube induced pharyngolaryngeal spasms, a Witzel type feeding gastrostomy was fashioned. 은 The severity of the tetanus was apparent, not only from the $\omega$ spasm frequency, but by the appearance of severe 

using Intrathecal Human Tetanus Immunoglobulin and External Fixation

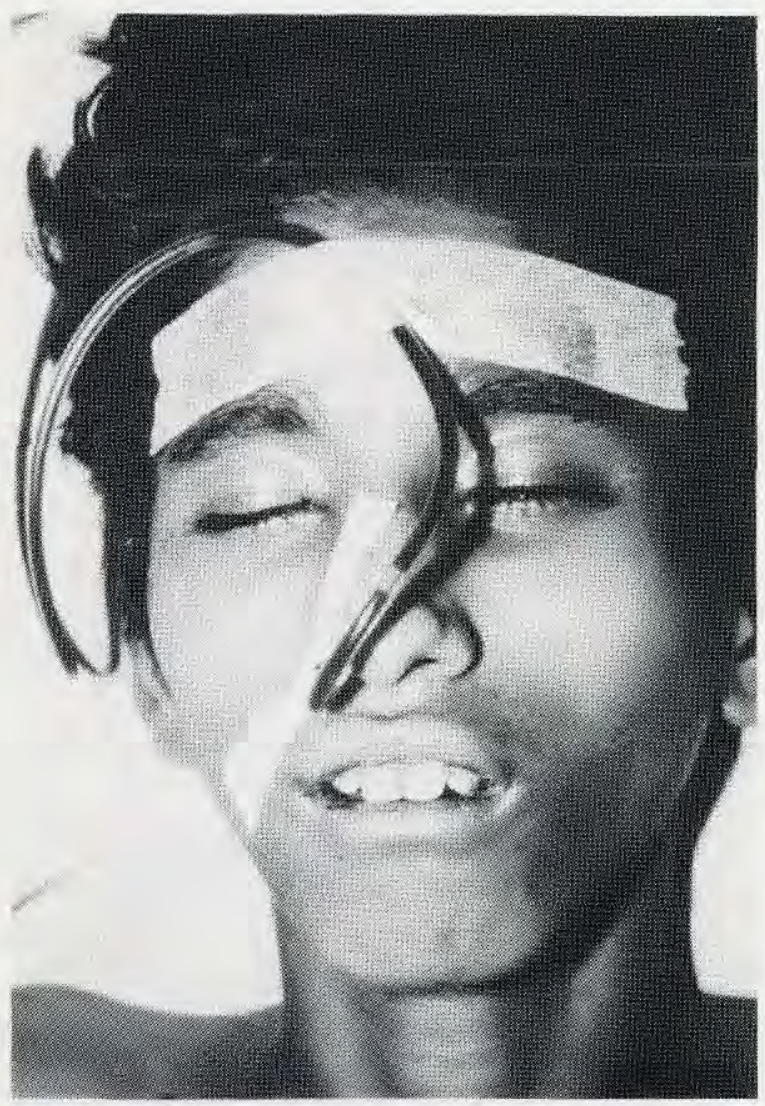

Fig. 1. Risus Sardonicus

autonomic dysfunction in the form of sweating, cold peripheries and paroxysmal tachycardia. The latter was controlled by propranolol $40 \mathrm{mg} \mathrm{t}$,d.s. via the gastrostomy. A total of three litres of water and electrolytes, and a liquidised diet of rice, lentils and chicken to the value of about 3000 calories a day, were administered through the feeding gastrostomy.

In an attempt to avoid the need for non-depolarising muscle relaxation, with its attendant nursing and logistical problems, 250 units of HTI were injected intrathecally under ketamine anaesthesia. Within five hours the sweating and peripheral vasospasm disappeared, and the spasm frequency, which had exceeded 20 per hour during the preceding 24 hours, fell to less than five per hour, and remained so during the subsequent two weeks.

Despite adequate control of the tetanus, major management difficulties were generated by the grossly infected compound fracture. The fracture became widely angulated and shortened with each spasm, causing further devitalisation. Strong conventional traction failed to overcome the powerful quadriceps contractions. Such was the severity and extent of infection that amputation was considered.
However, because of the immense problems facing 3 amputees in Nepal, it was decided to use rigid external $\stackrel{\otimes}{\Omega}$ fixation in an attempt to save the limb.

Pre-operatively, a further 250 units HTI were given in음 anticipation of massive toxin release during surgery. क्? Shortly after the commencement of surgery, the patient $\overrightarrow{\vec{F}}$ suddenly developed profound hypotension and $\stackrel{\oplus}{+}$ bradycardia, followed by cardiac asystole. Following resuscitation, the operation proceeded uneventfully. In the $\frac{\bar{\sigma}}{\bar{\omega}}$ absence of Schrang pins, REME-modified Denham pins $\frac{\text { D }}{\widetilde{D}}$ were used to achieve biplanar anterior and lateral fixation $\_$ through a Day frame (figure II). Post-operatively, recovery was complicated by bradycardia and hypotension which $\overrightarrow{0}$ responded to atropine and blood transfusion.

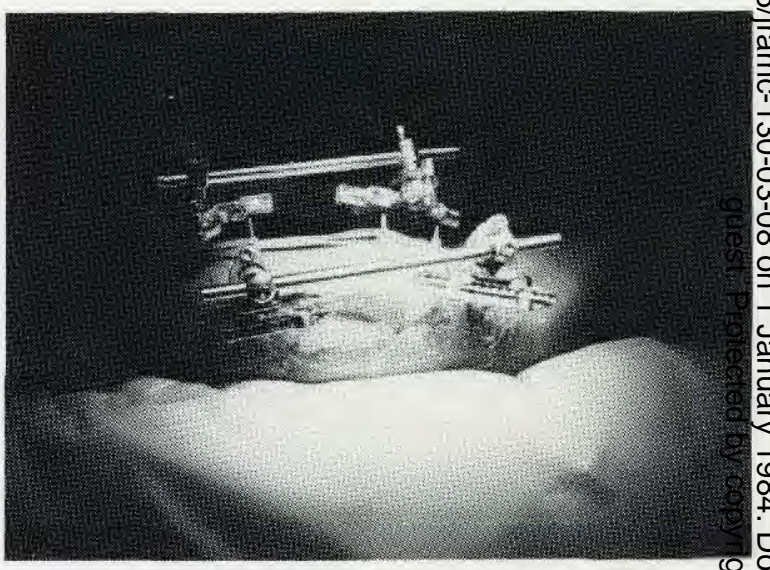

Fig. 2. External fixation device in position

Despite violent thigh muscle contractions, the biplanar $\frac{0}{\mathbb{D}}$ use of the Day frame provided sufficient stability to achieve $\stackrel{\varrho}{\vec{a}}$ rigid fixation of the fracture. Daily dressings under $\overrightarrow{\overrightarrow{0}}$ ketamine anaesthesia allowed rapid granulation of the 3 wound which was subsequently successfully grafted with? a 3:1 ratio meshed split skin graft. Bone union followed uneventfully. After three weeks, the intravenous medication was gradually reduced by decrements to zero. The lateral external fixation device was removed at six weeks and the anterior device at eight weeks post-fixation. $\mathbb{D}$

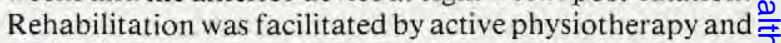
oral diazepam to overcome the residual muscular hypertonicity. Before discharge, a full course of tetanus 3 . toxoid immunisation was given.

\section{Discussion}

The complex management problems posed by this 은 patient stretched the medical and nursing resources of $\frac{D}{0}$ $\mathrm{BMH}$ Dharan to the limit. The incubation period of five days and onset time of less than one day forewarned of a $N$ very severe attack of tetanus with a high probability that $N$ turbocurarine would be required to control then spasms $^{2,3,4,5}$. The rapidly increasing spasm frequency and ${ }_{\sigma}^{\omega}$ the appearance of autonomic dysfunction suggested the 
likelihood of a fatal outcome. The spasms were not controlled by large doses of intravenous sedatives nor by the introduction of cortico-steroid therapy which has been shown to influence prognosis favourably ${ }^{3}$. Autonomic dysfunction, which is a dangerous complication of severe tetanus $^{6,7}$, appeared early and was only partially controlled by a combination of proranolol and chlorpromazine. Tracheostomy was done at the first sign of dysphagia, in anticipation of respiratory embarrassment, and greatly facilitated management.

A single dose of human tetanus immunoglobulin by the lumbar intrathecal route produced a dramatic improvement in what was a rapidly deteriorating situation, both with regard to a large reduction in spasm frequency, and the reversal of the autonomic dysfunction. The lumbar puncture, which was uneventful despite opisthotonos, was carried out under ketamine anaesthesia, in order to prevent spasms during the procedure. Intrathecal tetanus immunoglobulin was first suggested by Sherrington in $1917^{8}$, and more recently other groups have claimed benefit using horse antitetanus serum by this route $e^{9,10}$. The availability of safer human tetanus immunoglobulin has made the intrathecal route more attractive ${ }^{11}$. The effectiveness of intrathecal human tetanus immunoglobulin appears to be greater if given in the early stages of the disease ${ }^{11.12}$. A single dose of 250 units has been shown to be effective when used in addition to conventional therapy". In this case, it appeared to make a major lifesaving contribution.

The occurrence of hypotension followed by cardiac arrest whilst the fracture was being manipulated under general anaesthesia, highlights the dangers of surgery in patients with severe tetanus complicated by autonomic instability, particularly if beta blockade, in combination with chlorpromazine, an alpha blocker, is employed. The pharmacological autonomic paralysis exposes the patient to dangerous bradycardia and hypotension, occurring either spontaneously or in response to stimulation ${ }^{7,8,13}$.

Though using improvised pins, the external fixation device achieved a remarkable degree of stability, despite powerful thigh muscle contractions. Inadequately treated compound fractures in Nepal frequently result in a fatal outcome, due to severe infection or tetanus. The strength and simplicity of application of the frame makes it particularly suitable for use in such fractures, where stability is crucial to the management of the soft tissue of injury and preservation of the limb.

That this boy developed life-threatening tetanus after $\vec{s}$ being given penicillin and tetanus toxoid, illustrates the futility of this approach in severely wounded patients not previously immunised against tetanus ${ }^{5}$. A more $\frac{\omega^{5}}{\sigma}$ appropriate early treatment would have been adequate $\triangle$ surgical toilet and a single $1 \mathrm{ml}$ injection of human tetanus is immunoglobulin 250 units $^{14}$. Following this, and in all patients recovering from tetanus, a full course of tetanus toxoid should be given ${ }^{14}$.

\section{REFERENCES}

1 Eckman L. Tetanus, Prophylaxis and Treatment. Grune \& Stratton. London. 1963.

2 EDMONDSON R $\mathbf{S}$ and Flowers $\mathbf{M}$ W. Intensive Care in Tetanus: management, complications and mortality in $1 \otimes$ cases. Br Med J 1979; 1: 1401-1404.

3 Sanders R K M, Strong T $\mathbf{N} \&$ PEACOCK M C: the Treatment of Tetanus with Special Reference to Betamethasone. Trans $R$ Soc Trop Med Hyg 1969; 6 746-754.

4 Vakil B J, Armitage P and Laurence D R. Therapeut Trial of Intrathecal Human Tetanus Immunoglobulin Severe Tetanus: preliminary communications. Proceeding of the 4th International Conference on Tetanus, Daka Senegal 1975; p423-425.

5 Christie A B. Infectious Diseases Epidemiology and Cliniç̊ Practice. Churchill-Livingston Edinburgh. 3rd Ed 1988 p683-702.

6 KERR J H, et al. Involvement of the Sympathetic Nervot System in Tetanus. Lancet $1969 ; 2 ; 236-241$.

7 PRYS-ROBERTS C, et al. Treatment of Sympathetic Overactivity in Tetanus. Lancet 1969; 1: 542-546.

8 Sherrington C S. Observation with Antitoxin Serum in Monkeys. Lancet 1917; 1: 964-966.

9 SANDERS R K M, et al. Serum (horse) in the Treatment of Tetanus. Lancet 1977; 1: 974-977.

10 SANDERS R K M. The Treatment of Tetanus with Intrathecal Antitetanus Serum. Voluntary Health Organisation of India. $257 \mathrm{p} 1-7$.

11 GuPTA P S, et al. Human Tetanus Immunoglobulin in Early Tetanus. Lancet 1980; 11: 439-440.

12 GUPTA P S and KAPOOR R. Intrathecal Use of Human Tetanus Immunoglobulin. Clinician 1980; 44: 127-133.

13 Corbett J L, SPalding J M K and HarrisP J. Hypotension in Tetanus. Br Med J 1973; 2: 423-428.

14 Smith J W G. Laurence D R and Evans D G. Prevention of Tetanus in the Wounded. $\mathrm{Br}$ Med J 1975; 3: 453-455. 